\title{
Impacto dos papéis dos mais velhos na promoção da saúde em famílias com paramiloidose
}

\author{
Carla Roma Oliveira (iD 1,2, Álvaro Mendes 2,3 , \& Liliana Sousa ${ }^{1}$ \\ ${ }^{1}$ Departamento de Educação e Psicologia, Universidade de Aveiro, CINTESIS, Portugal \\ ${ }^{2}$ UnIGENe, IBMC Instituto de Biologia Molecular e Celular, i3S - Instituto de Investigação e Inovação em \\ Saúde, Universidade do Porto, Portugal \\ ${ }^{3}$ CGPP - Centro de Genética Preditiva e Preventiva, IBMC - Instituto de Biologia Molecular e Celular, i3S - \\ Instituto de Investigação e Inovação em Saúde, Universidade do Porto, Portugal
}

\begin{abstract}
Resumo: Os elementos mais velhos da família exercem papéis de promoção da saúde junto dos mais novos em doenças hereditárias como a paramiloidose. Contudo, escasseiam estudos que associem esses papéis ao impacto nas gerações mais novas. 0 objetivo deste estudo qualitativo exploratório é analisar o impacto dos papéis exercidos pelos mais velhos junto dos mais novos na promoção da saúde em famílias com paramiloidose. Adotou-se a Técnica dos Incidentes Críticos aplicada em entrevistas semiestruturadas. A amostra compreende 18 participantes, que relataram 76 incidentes. As entrevistas foram gravadas, transcritas e submetidas a análise de conteúdo. Os resultados sugerem que os impactos nos mais novos envolvem sobretudo capacitação para enfrentar a doença $(n=41)$ e dificultar/complexificar a experiência da doença $(n=11)$. 0 impacto dos papéis exercidos pelos mais velhos em famílias com paramiloidose é relevante, devendo ser considerado pelas equipas de saúde.
\end{abstract}

Palavras-chave: Amiloidose familiar; Doenças hereditárias; Gestão do risco; Promoção da saúde; Efeito de geração.

Impact of the older family members' roles in health promotion in families with paramyloidosis: Older family members play a role in health-promotion amongst the younger in hereditary diseases such as paramyloidosis. However, studies that associate these roles with the impact on the younger generations are scarce. The aim of this exploratory qualitative study is to analyze the impact of the roles played by elders in health promotion, in families with history of paramyloidosis. The study adopts the Critical Incidents Technique, applied by semi-structured interviews. The sample comprises 18 participants, who reported 76 incidents. The interviews were recorded, transcribed and analysed thematically. Main results suggest that the impacts on the youngest are: to empower them to cope with the disease $(n=41)$ and to hinder the experience of living with the disease $(n=11)$. The impact of the roles played by the elders in families with paramyloidosis is relevant to health management of the youngest.

Keywords: Familial amyloid polyneuropathy; Hereditary disorders; Risk management; Health promotion; Generation effect.

A paramiloidose é uma doença hereditária de início tardio que se manifesta tipicamente antes dos 40 anos (Conceição, 2012). Foi descrita pela primeira vez em 1952 (Andrade, 1952), sendo também conhecida pelas designações Polineuropatia Amiloidótica Familiar (PAF) de tipo I, PAF de tipo Português, ATTRV30M, doença de Corino de Andrade, ou popularmente por "doença dos pezinhos". É uma doença neurológica degenerativa, autossómica dominante, altamente incapacitante e fatal em 10 a 20 anos após o início dos sintomas (Conceição, 2012). Existem atualmente medidas terapêuticas, incluindo transplante hepático desde 1992, e medicação oral (tafamidis), desde 2012, que parecem retardar a evolução dos sintomas (Adams et al., 2016). A taxa de incidência em 2016 foi de 0.66/100,000 (Inês et al., 2018). Portugal regista a maior prevalência a nível mundial (22.93/100,000 habitantes adultos em 2016: 20\% de casos mundialmente), nomeadamente na zona costeira Norte (região de Vila do Conde e Póvoa de Varzim), embora existam focos relevantes no Chipre, Suécia, Itália e França (Inês et al., 2018).

Em Portugal está disponível, desde 2012, o diagnóstico genético pré-implantatório (DGPI), que permite a seleção e posterior transferência de embriões não-portadores para implantação no útero

\footnotetext{
${ }^{1}$ Morada para correspondência: Carla Roma Oliveira, Departamento de Educação e Psicologia, Universidade de Aveiro, Campus Universitário de Santiago, 3810-193, Aveiro, Portugal. Email: carlaromaoliveira@ua.pt
} 
(Valdrez et al., 2014). Desde 1985 está disponível o diagnóstico molecular, permitindo a realização do teste pré-sintomático (TPS) para determinar o estatuto perante a doença (portador ou não-portador) (Adams et al., 2016). Os TPS são dirigidos a pessoas em 50\% de risco, saudáveis, predizendo a possibilidade de desenvolverem a doença no futuro e o risco para a sua descendência. Realizam-se com aconselhamento genético (AG) e apoio psicossocial, no contexto do programa nacional de TPS para indivíduos em risco de doenças neurológicas de início tardio. 0 AG visa facilitar o processo de tomada de decisão sobre a realização ou não do TPS, incluindo apoio psicossocial na exploração do potencial impacto dos resultados do teste; e envolve a promoção da adaptação à informação genética e, no caso de portadores, do ajustamento à irreversibilidade da doença (Skirton et al, 2013).

A doença tem um caráter familiar e os membros de famílias com historial de doenças genéticas são geralmente confrontados com a doença noutros elementos da família desde idades precoces (Mendes \& Sousa, 2012). A comunicação na família sobre o risco genético é um fenómeno complexo influenciado por vários fatores, incluindo características individuais, funcionamento familiar e aspetos socioculturais (Gaff et al., 2007). Os membros destas famílias podem adotar diversos comportamentos de promoção da saúde e gestão de risco, ou seja, qualquer atividade para promover, proteger e manter a sua saúde $(W H O, 1998)$, ou, em alguns casos, dificultar a mesma.

A literatura tem destacado a influência da família na tomada de decisões sobre problemas de saúde individuais (Koehly et al., 2009; Mendes, 2012). O contributo das relações intergeracionais também tem sido destacado (Bengtson, 2001), em particular o papel dos elementos das gerações mais velhas perante doenças crónicas severas e hereditárias (Ashida \& Schafer, 2015; Mendes et al., 2011). Os estudos têm focado sobretudo cancros hereditários, sendo o papel dos mais velhos descrito em termos de: transmissão de informação sobre a doença na família; encorajamento à realização de rastreio genético; apoio emocional e instrumental (Ashida \& Schafer, 2015; Koehly et al., 2009; Mendes et al., 2011; Pantaleao et al., 2019, Oliveira et al., 2017a) . Por papéis entendem-se comportamentos exercidos pelos elementos mais velhos da família, de forma explícita ou implícita, consciente ou inconsciente, junto dos mais novos, em relação à experiência/vivência da doença na família (Oliveira et al., 2017a). Alguns estudos sugerem que os papéis dos mais velhos se associam ao aumento de bem-estar dos membros da família, facilitação da gestão individual e familiar da doença e aumento da adesão à realização dos testes genéticos (Ashida et al., 2011). Contudo, escasseiam estudos centrados em famílias com historial de paramiloidose ou outras doenças neurogenéticas com TPS disponível. Oliveira et al. (2017a, 2017b) analisaram os papéis exercidos pelos mais velhos junto dos mais novos da família, em termos de comportamentos de promoção da saúde e gestão do risco genético em famílias com paramiloidose. Identificaram quatro categorias de papéis: i) modelar comportamentos perante a doença, em que os mais velhos influenciam os mais novos através do seu comportamento, muitas vezes de forma não intencional; ii) (des)encorajar a realização do TPS; iii) apoiar os mais novos quando se confrontam com o resultado de portador e/ou na fase sintomática da doença; e iv) (não) informar sobre o risco ou a doença, em que os mais velhos transmitem (ou não) informação aos mais novos e/ou os incentivam (ou não) a procurar informação sobre a doença e tratamentos (Oliveira et al., 2017a). Contudo, fica por esclarecer o impacto destes papéis nos mais novos. Assim, o objetivo deste estudo é analisar o impacto percebido pelos mais novos sobre os papéis desempenhados pelos mais velhos da família. Os resultados darão contributos para intervenções de promoção da saúde e da gestão individual e familiar do risco de doenças hereditárias.

\section{MÉTODO}

Este estudo qualitativo e exploratório recorre à Técnica dos Incidentes Críticos (TIC) (Flanagan, 1954). A TIC tem sido utilizada em estudos na área da saúde (Warren-Jeanpiere et al. 2010) e em estudos com famílias (Sousa et al. 2010).

Trata-se de uma metodologia que permite recolher eventos que traduzem acontecimentos de vida e possibilita aceder à perspetiva individual sobre papéis significativos (Flanagan, 1954). Por "incidente" entende-se qualquer atividade humana observável, descrita de modo suficientemente completo, para permitir inferências acerca da ação. Para ser "crítico", o incidente deve representar um contributo significativo, positivo ou negativo, para o narrador (Flanagan, 1954; Francis, 2004). A TIC permite recolher eventos que ocorreram junto de pessoas-chave (que viveram a situação), para aprofundar conhecimento (Kemppainen, 2000).

\section{Instrumento}

Este estudo faz parte de um projeto mais alargado (Oliveira et al., 2017a; 2017b), em que numa primeira fase se identificaram os papéis exercidos pelos mais velhos em famílias com paramiloidose. Após solicitar 
Incidentes Críticos (IC) aos participantes, e depois de identificar o papel exercido pelo mais velho, solicitava-se que caraterizassem o impacto em si e/ou na sua família.

A TIC foi aplicada através de entrevistas telefónicas, com base num guião semiestruturado. No final recolheram-se os seguintes dados: sociodemográficos dos participantes e dos elementos da geração mais velha protagonistas do IC (idade, sexo e escolaridade); estatuto perante o risco/doença do participante e do protagonista (não portador; portador pré-sintomático; doente, considerando o tipo de tratamento: transplantado; medicação; sem tratamento).

\section{Procedimento}

Este estudo obteve aprovação da Comissão de Ética Humana do IBMC (2013-03). 0 método de amostragem foi intencional e não-probabilístico. 0 recrutamento foi mediado pela Associação Portuguesa de Paramiloidose (APP). 0 projeto foi apresentado à APP que aceitou colaborar e divulgou o estudo junto dos associados. Foram indicados critérios de inclusão (membros de famílias com historial de paramiloidose; idade $\geq 18$ anos; orientados no tempo e no espaço) e exclusão (ter doença do foro mental ou psicológico; abusar de substâncias; estar em situação clínica crítica) dos participantes. A APP facultou o contato de correio eletrónico ou contato da investigadora aos interessados em colaborar no estudo; ou enviou o contacto do interessado à investigadora para proceder aos contactos. Recorreu-se ainda à amostragem em bola de neve (pedir a cada participante que identificasse outro/s potencial/is participante/s) (Silverman, 2000). 0 primeiro contacto com os potenciais participantes ocorreu por via telefónica, em que a investigadora (primeiro autor) explicou objetivos, metodologia e colaboração solicitada. Todos aceitaram colaborar e foi agendada a entrevista (hora e data) de acordo com a conveniência dos participantes. Foi obtido o consentimento informado verbal, registado por gravação áudio, que incluía autorização de gravação da entrevista. A recolha de dados decorreu entre janeiro e abril de 2015. A duração média das entrevistas foi de 25 minutos (mínimo $=10$ minutos; máximo = 49 minutos). A recolha da amostra terminou com 18 participantes (76 IC relatados), quando os autores concordaram que o ponto de saturação (situação em que os dados já foram ouvidos durante a recolha) tinha sido atingido (Mason, 2010). Utilizou-se a avaliação inter-juízes para determinar a saturação: a primeira autora que conduziu as entrevistas anunciou quando considerou ter sido atingida a saturação; os dois outros autores leram independentemente os IC e impactos e indicaram a sua concordância.

\section{Participantes}

A amostra compreende 18 participantes, com idades entre os 18 e 65 anos (média $=42$ anos), residentes nas regiões Norte e Centro de Portugal continental. Em termos de grupo etário: $\leq 20$ anos = 1 participante; 21-30 anos $=2 ; 31-40$ anos $=3 ; 41-50$ anos= 9; 51-60 anos $=2 ; \geq 61$ anos $=1$. Dez são do sexo feminino. Quanto à escolaridade: $\leq 4$ anos de escolaridade $=1$ participante; 5 - 9 anos de escolaridade $=10 ; 10$ a 12 anos $=4$; ensino superior $=2 ; 1$ participante não responde. Em relação ao estatuto perante a doença, verificou-se que 11 são doentes ( 8 transplantados; 3 tomam medicação), 2 portadores pré-sintomáticos, 4 não-portadores e 1 ainda não sabe (não realizou TPS).

\section{Análise de Dados}

As entrevistas foram gravadas, ouvidas na totalidade e os IC e impactos transcritos e submetidos a análise de conteúdo (Miles \& Huberman, 1994), realizada por dois juízes independentes (primeiro e terceiro autores). Os juízes começaram por ler todos os IC (78) para decidir quais os elegíveis; neste processo foram eliminados 2 IC não relacionados com os objetivos do estudo (i.e., não relatavam uma ação de uma pessoa da geração mais velha junto da mais nova).

A análise dos dados iniciou-se com a criação e testagem do sistema de categorização. Cada juiz leu todos os IC e desenvolveu uma lista de categorias relativas aos papéis dos mais velhos e impactos nos mais novos. Depois reuniram-se para comparar e discutir propostas. Esse processo repetiu-se até chegarem a acordo. Em seguida cada juiz categorizou aleatoriamente 5 IC para confirmar a adequação do sistema de categorização; o sistema revelou-se adequado. Então, os juízes categorizaram de forma independente cada IC e respetivo impacto. Depois reuniram para analisar as (dis)concordâncias; a concordância entre juízes (valor calculado pela divisão de número de concordâncias pelo total de concordâncias e discordâncias) foi de $81,6 \%$ para os papéis $86,8 \%$ para os impactos, o que indica boa fiabilidade (Miles \& Huberman, 1994). Por fim, discutiram as discordâncias e esse processo conduziu à total concordância. E efetuou-se o cálculo das frequências (crosstabs) considerando papéis e impactos. 


\section{RESULTADOS}

Os 18 participantes relataram 76 IC (média de 4,3 por participante). Três participantes relataram um incidente (mínimo) e dois relataram 10 (máximo). Verificaram-se 24 não respostas (i.e., participantes identificaram os papéis, mas não indicaram impacto). A idade dos protagonistas mais velhos no momento do IC varia entre 32 e 78 anos e dos mais novos entre 12 e 49 anos (idade aproximada, pois nem sempre os participantes sabiam precisar a idade no momento do IC). A maioria dos incidentes ocorreu em ambientes familiares (19/76) ou em contextos de saúde, como hospitais ou consultório médico (9/76).

\section{Impactos}

Os impactos dos papéis da geração mais velha nos mais novos da família foram classificados em duas categorias principais: i) "capacitar para enfrentar a doença” (n=41), em que os papéis exercidos pelos mais velhos ajudam os mais novos a enfrentar a doença (sua ou de um familiar); e ii) "dificultar/complexificar a vivência da doença" $(n=11)$, quando os papéis dos mais velhos levam os mais novos a ter dificuldade em lidar com o sofrimento dos familiares mais velhos, sentir "raiva", não compreender e sentirem uma "herança pesada"; os mais novos portadores pré-sintomáticos também referem sentir-se assustados, por pensarem no que lhes vai acontecer no futuro e não estarem preparados para enfrentar a doença e os tratamentos (sobretudo, o transplante).

A categoria "capacitar" envolve três subcategorias:

i) "sentir-se preparado para enfrentar a doença" $(n=17)$, através de "exemplos "positivos" dos mais velhos (como participar em ensaios clínicos), e "negativos" (por exemplo, os mais velhos recusarem realizar os tratamentos disponíveis, levando os mais novos, que assistem à sua deterioração física, a tomar decisões diferentes.

ii) "sentir apoio" (n=12), os mais novos sentem que têm o apoio dos mais velhos para qualquer necessidade, ou veem-nos a apoiar outros elementos da família doentes.

iii) "tomar consciência da doença" ( $n=12)$, os mais novos sentem que o papel dos mais velhos foi determinante para terem efetuado o diagnóstico molecular (através do TPS) e terem aprofundado o seu conhecimento sobre a doença (sintomas, evolução e acompanhamento clínico disponível).

O impacto capacitar ocorre sobretudo entre mais velhos portadores sintomáticos e mais novos que desconheciam o seu estatuto genético no momento em que ocorreu o IC $(n=14)$, sobretudo quando os mais velhos exercem os papéis: "modelar comportamentos" (6 IC), mas também quando encorajam os mais novos para realizarem o TPS (5 IC) e informam sobre a doença (3 IC); ocorre também entre mais velhos não portadores e mais novos portadores sintomáticos (6 IC), através do seu apoio (5 IC); ocorre ainda entre mais velhos e mais novos, ambos portadores sintomáticos (6 IC), através do papel "modelar" (5 IC ) e "apoiar" (1 IC); e entre mais velhos não portadores e mais novos que desconheciam o seu estatuto genético (5 IC), em que os mais velhos encorajam a realização TPS (3 IC), informam (1 IC) e apoiam os mais novos (1IC).

Os protagonistas mais velhos são maioritariamente mulheres (51/76), sobretudo mães, mas também avós, tias e primas em que 24/52 IC correspondem ao impacto capacitar e 10/52 a dificultar. Capacitar resulta sobretudo de papéis exercidos entre mãe e filha/o (17 IC), pai e filha/o (12 IC) e mais pontualmente envolve tios/as-sobrinho/a, avós, netos/as e primas.

Quanto ao impacto dificultar, os dados indicam que ocorre sobretudo entre mais velhos portadores sintomáticos e mais novos que desconheciam o seu estatuto genético (5 IC), sobretudo através do papel "modelar comportamentos" (4 IC) e "informar" (1 IC); e entre mais velhos portadores sintomáticos e mais novos não portadores através do papel "modelar" (2 IC).

Os dados indicam que o impacto dificultar ocorre sobretudo entre mãe e filha/o (8 IC), 1 IC entre pai e filha e 2 IC entre tia e sobrinho/a.

Quanto ao local de ocorrência dos incidentes verificou-se que a maior parte (19/76 IC) decorriam em ambientes de família e em contextos de cuidados de saúde como o hospital ou consultório médico.

\section{Impacto e Papéis}

Os papéis relatados foram associados aos impactos nos mais novos (Tabela 1). "Capacitar" associa-se sobretudo ao papel dos mais velhos "modelar comportamentos perante a doença" (n=20), seguindo-se "apoiar" (n=8), "(des) encorajar realizar o TPS", (n=8) e "informar sobre o risco/doença” (n=5).

A subcategoria "sentir-se preparado para enfrentar a doença" associa-se principalmente ao papel "modelar os comportamentos perante a doença" (n=14), nas subcategorias "normalizar a vivência da doença" (n=8) e ser "testemunho vivo da doença" (n=6). Os mais velhos ajudam os mais novos (sobretudo os portadores assintomáticos e os que desconhecem o seu estatuto perante a doença) pela forma como 
continuam a cuidar-se apesar dos sintomas (testemunho vivo da doença), e como dão apoio e se preocupam com os outros, apesar de doentes (normalizar a vivência da doença).

"A minha mãe [portadora sintomática] tinha uma particularidade que era o facto de se dar muito aos outros. Ela tratou sempre da minha tia (...), do meu avô, sempre foi muito voltada para os outros e muito pouco centrada em si. Isso ajudou brutalmente (...) porque a minha mãe não valorizava a doença no sentido em que procurava pensar nos outros. (...) Isto fazia-a descentrar-se da doença e não valorizar." [mulher, portadora assintomática, 47]

Tabela 1. Impactos dos papéis dos mais velhos nos mais novos

\begin{tabular}{|c|c|c|c|c|}
\hline \multirow[t]{2}{*}{ Papéis } & \multicolumn{4}{|c|}{ Impactos $(n=52$ IC $)$} \\
\hline & \multicolumn{3}{|c|}{ Capacitar } & Dificultar \\
\hline & $\begin{array}{l}\text { Sentir } \\
\text { apoio }\end{array}$ & $\begin{array}{l}\text { Consciência da } \\
\text { doença }\end{array}$ & $\begin{array}{l}\text { Sentir-se } \\
\text { preparado }\end{array}$ & \\
\hline $\begin{array}{l}\text { Modelar comportamentos perante a } \\
\text { doença }\end{array}$ & 4 & 2 & 14 & 8 \\
\hline Normalizar a vivência da doença & 4 & 1 & 8 & 0 \\
\hline Dramatizar a vivência da doença & 0 & 0 & 0 & 1 \\
\hline Testemunho vivo da doença & 0 & 1 & 6 & 7 \\
\hline (des)Encorajar realizar TPS & 1 & 6 & 1 & $\mathbf{0}$ \\
\hline Encorajar & 0 & 4 & 1 & 0 \\
\hline Apoiar qualquer decisão & 0 & 1 & 0 & 0 \\
\hline Acompanhar na realização do TPS & 1 & 1 & 0 & 0 \\
\hline \multicolumn{5}{|l|}{ Desencorajar } \\
\hline Apoiar & 7 & 1 & $\mathbf{0}$ & 2 \\
\hline Apoio emocional (resultado TPS) & 2 & 1 & 0 & 0 \\
\hline Aconselhar sobre decisões pessoais & 0 & 0 & 0 & 2 \\
\hline $\begin{array}{l}\text { Apoio emocional e instrumental na } \\
\text { doença }\end{array}$ & 3 & 0 & 0 & 0 \\
\hline Apoiar decisão de tratamentos & 2 & 0 & 0 & 0 \\
\hline (não) Informar sobre risco/doença & $\mathbf{0}$ & 3 & 2 & 1 \\
\hline Informar & 0 & 2 & 1 & 0 \\
\hline Incentivar procurar informação & 0 & 1 & 1 & 1 \\
\hline \multicolumn{5}{|l|}{ Não falar (silenciar) } \\
\hline Total & 12 & 12 & 17 & 11 \\
\hline
\end{tabular}

A subcategoria "sentir apoio" relaciona-se sobretudo com o papel "apoiar" (n=7), nas subcategorias: i) "apoio emocional e instrumental na doença" $(\mathrm{n}=3)$

Ela [mãe não portadora] (...) sempre esteve do meu lado, sempre me apoiou, sempre cuidou de mim ou leva-me aqui, leva-me ali. Se eu estivesse mais doente, ela nunca falhou. [Homem, portador sintomático, 49 anos]

ii) “apoio emocional (resultado TPS)" (n=2)

"Quando cheguei a casa depois de saber o resultado [TPS], ele [pai não portador] abriu a porta e eu estava cabisbaixo, encharcado em lágrimas e ele agarrou-se a mim e deu-me um abraço muito grande (...) levantou-me a cabeça e deu-me um beijinho e disse "ó filho estamos juntos, vamos tentar ultrapassar isto da melhor maneira". (...) Este dia foi muito marcante pela negativa, mas também pela positiva. (...) Eu saí de casa algo moralizado e motivado (...) foi muito importante, foi determinante ter tido esse momento" [Homem, portador sintomático, 49 anos]

iii) "apoiar decisão de tratamentos" (n=2)

"O meu filho [portador sintomático] foi inscrito para fazer o transplante. Só que naquela altura (...) havia lista de espera, eram muitos, (...) A doença começou a avançar (...) e eu tive de tentar mexer-me (...) não ia deixar o meu filho numa cadeira de rodas, (...) não podia ser. Foi quando eu tentei na Inglaterra, na Alemanha, na França e depois descobri um médico em Espanha (...) eu consegui no espaço de um mês (...). [0 transplante] Foi há 8 anos, está aí bem, está a trabalhar e com responsabilidades" [Homem, não portador, 65 anos] 
A subcategoria "sentir apoio" surge associada ao papel "modelar os comportamentos perante a doença" (n=4), através de "normalizar a vivência da doença" (n=4)

"Ele [pai não portador] lidou bem quando a minha mãe soube que tinha a doença. Connosco [filhos que receberam o resultado de portadores] foi: "olha paciência, é seguir em frente, tratar como a vossa mãe tratou"!" [Mulher, portadora sintomática, 32 anos]

"Tomar consciência da doença" associa-se a "des/encorajar realizar o TPS" $(n=6)$, sobretudo através de "encorajar" (n=4):

"Foi quando a minha mãe [não portadora] disse: "tu tens a mesma doença que o teu pai". (...) Ela disse que eu estava a passar as mesmas coisas que o meu pai passou e depois mandou-me fazer o teste. (...) Eu tinha 24 anos. (...) Eventualmente, eu acabava por ir fazer os testes. Podia demorar mais 6 meses ou um ano, mas..." [Homem, portador sintomático, 49 anos]

0 impacto "dificultar a vivência da doença" relaciona-se principalmente com "modelar os comportamentos perante a doença" ( $\mathrm{n}=8$ ) sendo "testemunho vivo da doença" $(\mathrm{n}=7)$.

"Todos os dias eram dias muito penosos, muito dolorosos porque as queixas e a doença em si eram realmente complicadas (...). Pensei que era a perda da dignidade total da pessoa, o deixar de fazer certos movimentos, a procura do olhar da minha mãe [portadora sintomática] à procura de nós e não nos ver, não nos encontrar, penso que isso foi dos momentos mais difíceis que testemunhei com ela. E no Porto, quando íamos às consultas era extremamente difícil, ainda hoje é, encontrar pessoas que também têm a doença. (...) Para mim é [difícil] porque ainda não tenho [sintomas], não é? E quando lá vou revejo tudo isto e penso que me vai acontecer a mim também. É sempre difícil esse impacto de contactar com a doença." [Mulher, portadora assintomática, 42 anos]

Surge ainda em situações em que os mais velhos "aconselham sobre decisões pessoais" $(\mathrm{n}=2)$.

"O meu pai [não portador] achava que eu não ia ter filhos (...) ele nunca falou comigo diretamente, mas uma minha cunhada ficou muito admirada quando eu lhe dei a notícia que estava grávida e queria engravidar porque uma vez o meu pai em conversa com ela lhe terá dito que eu não ia ter filhos, que não podia, mas não foi conversa que ele tivesse comigo. (...) Ele na altura não reagiu assim muito bem, mas depois também lhe expliquei o que estava a fazer para tentar que a [nome da filha] não tivesse a doença. (...) não era uma decisão fácil (...). Claro que não era uma grande ajuda o meu pai pensar isso, mas eu também só soube depois de tomar a decisão de engravidar, não me afetou, mas... [Mulher, portadora assintomática, 42 anos]

Em geral, os papéis associam-se a capacitar ou a dificultar. Contudo, duas subcategorias dos papéis surgem associados simultaneamente aos dois impactos. "Ser testemunho vivo da doença" (papel: modelar comportamentos perante a doença) apresenta 7 IC associados a "capacitar" e 7 IC a "dificultar". E "incentivar procurar informação" (papel: informar sobre a doença), tem 2 IC associados a "capacitar", em que a informação ou o apoio na sua procura ajudou os mais novos a tomarem consciência do que é a doença e a prepararem-se para a enfrentar; mas também 1 IC a "dificultar", quando o confronto com a informação numa idade jovem e numa fase de tomada de consciência sobre a doença, parece "chocar" os mais novos.

"Depois de ela [mãe portadora sintomática] ter descoberto a doença (...) estivemos a ver noticias e fotos sobre a doença e realmente a progressão não é agradável de se ver. Penso que a parte que mais me chocou foi pensar que a minha mãe poderia ficar dessa maneira". [Homem, estatuto desconhecido, 18 anos]

\section{DISCUSSÃO}

Nas famílias com paramiloidose os mais velhos exercem papéis (Oliveira et al., 2017a), com impacto na forma dos mais novos enfrentarem o seu estatuto perante a doença ou os sintomas da doença. 0 impacto ocorre sobretudo na capacitação do enfrentamento da doença pelos elementos da geração mais nova; e com menor frequência, no sentido de o dificultar. Capacitar envolve sentir-se preparado para no futuro enfrentar a doença em si e/ou em familiares próximos; sentir apoio para as dificuldades que a doença envolve; e tomar consciência da doença, i.e., saber o que envolve ter a doença.

Estes resultados enquadram-se nos desafios psicossociais das doenças hereditárias para indivíduos e famílias (Rolland \& Williams, 2005) (tomada de consciência sobre a doença, sentir-se preparado para a possível ocorrência de sintomas e sentir-se apoiado na vivência da doença, após o início dos sintomas em si ou num familiar). 0 impacto capacitar e as suas subcategorias resultam principalmente de papéis exercidos por elementos mais velhos portadores sintomáticos (em alguns casos também não portadores) que "modelam comportamentos", normalizando a experiência e/ou sendo "testemunho vivo" da doença (os portadores sintomáticos). Estes impactos observam-se sobretudo quando os mais velhos afetados permitem aos mais novos conhecerem a doença e compatibilizam a gestão desta com outras funções familiares, sociais e profissionais; e, quando os mais velhos saudáveis cuidam dos membros da família doentes (Oliveira et al., 2017a, 2017b). Assim, os mais novos (sobretudo portadores sintomáticos ou que desconhecem o seu estatuto perante a doença) sentem-se capacitados, por se sentirem mais preparados 
para enfrentar a doença, pois têm modelos de papel; mas também porque sabem que terão apoio de membros da família se/quando a doença se manifestar; além disso tomam consciência do que é a doença. Estes dados parecem corroborar resultados de estudos que associam o conhecimento e experiência prévia com a paramiloidose a maior preparação para enfrentar a condição de risco (Paneque et al., 2009).

Os mais novos também se sentem capacitados quando os mais velhos os encorajam a realizar o TPS e lhes dão informação e apoio instrumental e/ou emocional. Estes resultados são consistentes com estudos em que o apoio (instrumental, emocional e à tomada de decisão) e encorajamento (para realizar o TPS) dos mais velhos se associam a maior bem-estar e coesão familiar, facilitação da gestão da doença e aumento de comportamentos de saúde e gestão do risco genético (Ashida et al., 2011; Koehly et al., 2009; Pantaleao, 2009). São compatíveis com as exigências e desafios colocados a indivíduos e famílias ao longo da doença (Rolland \& Williams, 2005). E reforçam a importância da reciprocidade intergeracional através do envolvimento dos mais velhos enquanto recurso para as famílias (Bengtson, 2001; Ernsth-Bravell et al., 2016; Lowenstein et al., 2007).

Os mais novos assistem à progressão da doença nos mais velhos, através do seu "testemunho vivo" (Oliveira et al. 2017a), e aprendem a conhecer a paramiloidose através dos sintomas, incapacidade, reações, sofrimento e "perda de dignidade" dos mais velhos. Em simultâneo, assistem à reorganização e reajustamento familiar em torno dos cuidados. 0 impacto "dificultar" a gestão/vivência da doença surge associado ao papel "modelar", sobretudo "ser testemunho vivo da doença"; papel "apoiar", "aconselhar sobre decisões da vida pessoal". Estudos de Lopes et al. (2018) sugerem que os elementos destas famílias convivem desde cedo com a doença e a morte precoce de algum dos progenitores. Alguns tornam-se os cuidadores principais destes familiares doentes. Para alguns dos mais novos, a exposição a estas circunstâncias e o conhecimento do caráter hereditário da doença, associam-se a maior vulnerabilidade no enfrentamento da doença na idade adulta. E também veicula perceção de maior dificuldade em cumprirem objetivos normativos, como criarem a própria família e terem filhos.

O papel "modelar", através da subcategoria "testemunho vivo da doença" tem impacto ambíguo, pois associa-se a "capacitar" (sentir-se preparado) e a "dificultar". Este papel é exercido de forma implícita (Oliveira et al., 2017a; 2017b)), ou seja, deriva de "observações não comentadas" (Palmquist et al., 2010) a que os mais novos têm acesso, provavelmente desde cedo, sem apoio dos mais velhos na gestão e integração desta informação. Assim, enquanto alguns dos mais novos integram esta informação sobre a doença e sentem-se mais preparados; outros deparam-se com sofrimento e dor associados à doença, sentindo dificuldade de enfrentamento. Estes resultados reforçam a importância de atender à história familiar quando se analisam os comportamentos atuais dos indivíduos e famílias, pois pode ajudar a prever e explicar o estilo de enfrentamento e adaptação à doença (Rolland \& Williams, 2005).

Os impactos resultam de relações entre elementos de duas (por vezes três) gerações, com relações de consanguinidade e envolvendo gerações contíguas. São principalmente as mulheres (sobretudo mães) a assumirem os diversos papéis junto de outras mulheres mais novas (em particular, filhas); este papel das mulheres como elos de ligação na família e cuidadoras tem sido evidenciado em diversos estudos sobre relações familiares e cuidados informais (Pantaleao et al., 2019; Routh et al., 2019; Vicente \& Sousa, 2010).

Os resultados deste estudo sugerem que o impacto dos papéis exercidos pelos elementos mais velhos de famílias com paramiloidose é relevante na gestão da saúde dos mais novos. Em geral, o impacto dos mais velhos é sobretudo percecionado pelos mais novos como capacitador e empoderador. Contudo, também ocorre no sentido de dificultar a vivência da doença, sendo relevante os profissionais de saúde ajudarem os mais novos a integrar eventuais observações não comentadas, nomeadamente as sentidas como pressão ou coerção para a tomada de decisões sobre a realização de TPS, relacionamentos e opções reprodutivas.

Atendendo a que as crenças familiares sobre as doenças se desenvolvem a partir da partilha de experiências e tradições intra/entre gerações (Mendes, 2012), parece reforçada a importância de se adotar uma perspetiva intergeracional na análise da gestão da saúde de indivíduos com história familiar de doenças hereditárias. Nesta linha, os mais velhos poderão ser um recurso importante para as gerações mais novas (Ashida et al., 2011; Mendes, 2012) ou até parceiros "escondidos" dos profissionais de saúde (Crisp et al., 2014), que deverão avaliar a pertinência do seu envolvimento junto dos mais novos.

\section{Limites e Perspetivas de Pesquisa}

A principal limitação deste estudo é o tamanho reduzido da amostra, apesar da saturação dos dados. Além disso, metade dos participantes situaram-se na faixa etária dos 41-50 anos (n=9), o que limita a perceção dos elementos mais novos sobre os impactos. Os participantes tiveram dificuldade em identificar os impactos (24 não respostas). Uma amostra maior e mais diversificada permitiria compreender melhor os impactos e analisar as relações de parentesco. Estudos posteriores poderiam incluir a perspetiva de elementos da família que não fizeram o TPS; recolher e comparar a perspetiva dos profissionais; estudar o 
impacto dos papéis dos mais velhos em famílias com outras doenças hereditárias; e explorar as situações em que um papel exercido pelos mais velhos resulta em impactos diferentes junto dos mais novos.

\section{CONCLUSÃO}

Os papéis desempenhados pelos elementos mais velhos de famílias com história de paramiloidose têm impacto nos elementos mais novos. Estes impactos ocorrem no sentido de capacitar (mais frequente) e (menos vezes) dificultar o processo de gestão e enfrentamento da doença. Assim, os mais velhos estão envolvidos na gestão do risco e da doença junto dos mais novos e a compreensão do impacto dos papeis que desempenham é relevante para compreender a história e as estratégias familiares de adaptação e gestão da doença e identificar as suas forças e vulnerabilidades (Rolland \& Williams, 2005). Estes resultados deverão ser considerados nas intervenções de promoção da saúde e da gestão individual e familiar do risco de doenças hereditárias. 0 envolvimento dos mais velhos da família pelos serviços e profissionais de saúde pode contribuir para a prática clínica, nomeadamente no apoio psicossocial a portadores em protocolos de teste pré-sintomático e do acompanhamento clínico multidisciplinar; além disso, enquadra-se na mudança dos paradigmas de saúde que sugerem maior partilha de responsabilidade pelos cuidados entre cidadãos/familiares e profissionais.

\section{REFERÊNCIAS}

Adams, D., Suhr, O. B., Hund, E., Obici, L., Tournev, I., Campistol, J. M., Slama, M. S., Hazenberg, B. P., \& Coelho, T. (2016). First European consensus for diagnosis, management, and treatment of transthyretin familial amyloid polyneuropathy. Current Opinion in Neurology, 29(Supplement 1), S14-S26. https://doi.org/10.1097/wco.0000000000000289

Andrade, C. (1952). A peculiar form of peripheral neuropathy. Brain, 75(3), 408-427.

Ashida, S., Hadley, D. W., Goergen, A. F., Skapinsky, K. F., Devlin, H. C., \& Koehly, L. M. (2011). The importance of older family members in providing social resources and promoting cancer screening in families with a hereditary cancer syndrome. The Gerontologist, 51(6), 833-842. http://doi.org/10.1093/geront/gnr049

Ashida, S., \& Schafer, E. J. (2015). Family health information sharing among older adults: reaching more family members. Journal of Community Genetics, 6(1), 17-27. http://doi.org/10.1007/s12687-0140197-x

Bengtson, V. L. (2001). Beyond the nuclear family: The increasing importance of multigenerational bonds: The burgess award lecture. Journal of Marriage and Family, 63(1), 1-16.

Conceição, I. (2012). Clinical features of TTR-FAP in Portugal. Amyloid, 19(Sup 1), 71-72. http://doi.org/10.3109/13506129.2012.673184

Crisp, L. N., Berwick, D., Kickbusch, I., Bos, W., Antunes, J., Barros, P., \& Soares, J. (2014). Um futuro para a saúde: todos temos um papel a desempenhar. Fundação Calouste Gulbenkian. http://www.gulbenkian.pt/mediaRep/gulbenkian/files/institucional/FTP_files/pdfs/FuturodaSau de2014/RelatorioFuturodaSaudePT2014/index.html

Ernsth-Bravell, M., Jegermalm, M., Fransson, E., \& Zarit, S. (2016). Reciprocal patterns of support of very old people and their families. The Gerontologist, 56, 215-215. https://doi.org/10.1093/geront/gnw162.847

Flanagan, J. C. (1954). The critical incident technique. Psychological Bulletin, 51(4), 327-358. http://doi.org/10.1037/h0061470

Francis, D. I. (2004). Reconstructing the meaning given to critical incidents in nurse education. Nurse Education in Practice, 4(4), 244-249. http://doi.org/10.1016/j.nepr.2004.01.001

Gaff, C. L., Clarke, A. J., Atkinson, P., Sivell, S., Elwyn, G., Iredale, R., Thornton, H., Dundons, J., Shaw, C., \& Edwards, A. (2007). Process and outcome in communication of genetic information within families: A systematic review. European Journal of Human Genetics, 15(10), 999. http://doi.org/10.1038/sj.ejhg.5201883

Inês, M., Coelho, T., Conceicão, I., Duarte-Ramos, F., de Carvalho, M., \& Costa, J. (2018). Epidemiology of transthyretin familial amyloid polyneuropathy in Portugal: A nationwide study. Neuroepidemiology, 51(3-4), 177-182. http://doi.org/10.1159/000490553

Kemppainen, J. K. (2000). The critical incident technique and nursing care quality research. Journal of Advanced Nursing, 32(5), 1264-1271. http://doi.org/10.1046/j.1365-2648.2000.01597.x

Koehly, L. M., Peters, J. A., Kenen, R., Hoskins, L. M., Ersig, A. L., Kuhn, N. R., Loud, J. T., \& Greene, M. H. (2009). Characteristics of health information gatherers, disseminators, and blockers within families at risk 
of hereditary cancer: implications for family health communication interventions. American Journal of Public Health, 99(12), 2203-2209. http://doi.org/10.2105/AJPH.2008.154096

Lopes, A., Sousa, A., Fonseca, I., Branco, M., Rodrigues, C., Coelho, T., Sequeiros, J., \& Freitas, P. (2018). Life paths of patients with transthyretin-related familial amyloid polyneuropathy Val30Met: a descriptive study. Journal of Community Genetics, 9(1), 93-99. http://doi.org/10.1007/s12687-0170338-0

Lowenstein, A., Katz, R., \& Gur-Yaish, N. (2007). Reciprocity in parent-child exchange and life satisfaction among the elderly: a cross-national perspective. Journal of Social Issues, 63(4), 865-883. https://doi.org/10.1111/j.1540-4560.2007.00541.x

Mason, M. (2010). Sample size and saturation in PhD studies using qualitative interviews. Forum qualitative Sozialforschung/Forum: Qualitative Social Research, 11(3), Art. 8. http://nbnresolving.de/urn:nbn:de:0114-fqs100387

Mendes, Á. (2012). Doenças hereditárias, aconselhamento genético e redes familiares e sociais: da ética intergeracional ao papel dos mais velhos. Revista Kairós: Gerontologia, 15, 199-216.

Mendes, A., Santos, T., \& Sousa, L. (2011). Experiencing genetic counselling for hereditary cancers: The client's perspective. European Journal of Cancer Care, 20(2), 204-211. http://doi.org/ 10.1111/j.1365-2354.2010.01201.x

Mendes, A., \& Sousa, L. (2012). Families' experience of oncogenetic counselling: accounts from a heterogeneous hereditary cancer risk population. Familial Cancer, 11(2), 291-306. http://doi.org/10.1007/s10689-012-9514-x

Miles, M. B., Huberman, A. M., Huberman. (1994). Qualitative data analysis: An expanded sourcebook. Sage.

Oliveira, C. R., Mendes, Á., Sousa, L. (2017a). Promoção da Saúde em famílias com paramiloidose: papéis dos mais velhos junto dos mais novos. Cadernos de saúde Pública, 33(5), e00185515. http://doi.org/10.1590/0102-311X00185515

Oliveira, C. R., Mendes, Á., Sousa, L. (2017b). From older to younger: intergenerational promotion of health behaviours in Portuguese families affected by familial amyloid polyneuropathy. European Journal of Human Genetics, 25, 687-693. http://doi.org/10.1038/ejhg.2017.40

Palmquist, A. E., Koehly, L. M., Peterson, S. K., Shegog, M., Vernon, S. W., \& Gritz, E. R. (2010). “The cancer bond": exploring the formation of cancer risk perception in families with Lynch syndrome. Journal of Genetic Counseling, 19(5), 473-486. http://doi.org/10.1007/s10897-010-9299-8

Paneque, M., Lemos, C., Sousa, A., Velazquez, L., Fleming, M., \& Sequeiros, J. (2009). Role of the disease in the psychological impact of pre-symptomatic testing for SCA2 and FAP ATTRV30M: Experience with the disease, kinship and gender of the transmitting parent. Journal of Genetic Counseling, 18(5), 483493. http://doi.org/10.1007/s10897-009-9240-1

Pantaleao, A., Young, J. L., Epstein, N. B., Carlson, M., Bremer, R. C., Khincha, P. P., Peters, J. A., Greene, M. H., Roy, K., Achatz, M. I., Savage, S. A., \& Werner-Lin, A. (2019). Family health leaders: Lessons on living with li-fraumeni syndrome across generations. Family Process, 59(4), 16481663.http://doi.org/10.1111/famp.12497

Rolland, J. S., \& Williams, J. K. (2005). Toward a biopsychosocial model for 21st-century genetics. Family Process, 44(1), 3-24. http://doi.org/10.1111/j.1545-5300.2005.00039.x

Routh, B., Hurt, T., Winham, D., \& Lanningham-Foster, L. (2019). Family legacy of diabetes-related behaviors: An exploration of the experiences of African American parents and adult children. Global Qualitative Nursing Research, 6, 1-12. https://doi.org/10.1177/23333936198523

Silverman, D. (2000). Doing qualitative research: A practical handbook. SAGE.

Skirton, H., Goldsmith, L., Jackson, L., \& Tibben, A. (2013). Quality in genetic counselling for presymptomatic testing-clinical guidelines for practice across the range of genetic conditions. European Journal of Human Genetics, 21(3), 256. http://doi.org/10.1038/ejhg.2012.174

Sousa, L., Silva, A. R., Santos, L., \& Patrão, M. (2010). The family inheritance process: Motivations and patterns of interaction. European Journal of Ageing, 7(1), 5-15. http://doi.org/10.1007/s10433-0100139-3

Valdrez, K., Silva, S., Coelho, T., \& Alves, E. (2014). Awareness and motives for use and non-use of preimplantation genetic diagnosis in familial amyloid polyneuropathy mutation carriers. Prenatal Diagnosis, 34(9), 886-892. http://doi.org/10.1002/pd.4388

Vicente, H. T., \& Sousa, L. (2010). Funções na família multigeracional: Contributo para a caracterização funcional do sistema familiar multigeracional. Psychologica (53), 157-181.

Warren-Jeanpiere, L., Miller, K. S., \& Warren, A. M. (2010). African American women's retrospective perceptions of the intergenerational transfer of gynecological health care information received from mothers: Implications for families and providers. Journal of Family Communication, 10(2), 81-98. http://doi.org/10.1080/15267431003595454 
Impacto dos papéis dos mais velhos na paramiloidose

WHO (1998). Health promotion glossary. Geneva: World Health Organization.

Historial do artigo

Recebido $\quad 02 / 12 / 2020$

Aceite $\quad 19 / 07 / 2021$

Publicado 20/12/2021 